\title{
A National Survey of Pediatric Intensive Care Units in Pakistan
}

\author{
Anwarul Haque, ${ }^{1}$ Laila A. Ladak, ${ }^{1}$ Muhammad H. Hamid, ${ }^{2}$ Sadiq Mirza, ${ }^{3}$ \\ Naveed R. Siddiqui, ${ }^{1}$ and Zulfiqar A. Bhutta ${ }^{1}$ \\ ${ }^{1}$ Department of Pediatrics and Child Health and Department of Clinical Trial Unit, Aga Khan University Hospital, \\ Karachi 74800, Pakistan \\ ${ }^{2}$ Institute of Children Hospital, Lahore, Pakistan \\ ${ }^{3}$ Abbasi Shaheed Hospital, Karachi, Pakistan
}

Correspondence should be addressed to Anwarul Haque; anwar.haq@aku.edu

Received 30 April 2013; Revised 8 August 2013; Accepted 5 September 2013; Published 5 January 2014

Academic Editor: Ricardo Rivera-Fernández

Copyright (C) 2014 Anwarul Haque et al. This is an open access article distributed under the Creative Commons Attribution License, which permits unrestricted use, distribution, and reproduction in any medium, provided the original work is properly cited.

Purpose. To describe the structure, staffing resources, equipment, academic activities, and characteristics of pediatric population of pediatric intensive care units across the country. Material \& Method. This was a prospective, descriptive, and observational survey of pediatric intensive care units from January to December 2009 across Pakistan. A questionnaire survey was emailed to director of each unit. Results. 16 PICUs were participated in this survey (100\% response rate). A total of units with 155 beds were identified (1.1 bed /500,000 children). Regarding the categories, 12 (75\%) were medical, 3 (19\%) were pure cardiac intensive care units, and one unit (6\%) was combined multidisciplinary cardiothoracic unit. $13(81 \%)$ units were in public sector as compared to 3 (19\%) were in private sector. The mean unit size was 9.7 (range 4-28) beds. Twelve (75\%) units were located in three large cities. Only 3 (19\%) units have trained intensivist. $37 \%(6 / 16)$ had nurse to patient ratio of $1: 1-1: 2$ while others had ratios of $1: 3-1: 5$ with all nurses specialized trained for pediatric intensive care units with bachelor degree or diploma in nursing. Only $50 \%$ had capacity for invasive monitoring. Conclusion. We found inadequacies in several aspects of PICUs in Pakistan including fewer PICUs, inadequate PICU beds, and lack of trained personal to look after critically ill pediatric population.

\section{Introduction}

Pediatric intensive care is recognized as a mature and established discipline in developed countries [1]. The availability of pediatric intensive care is a reflection of quality of country's pediatric medical care [2]. Pediatric intensive care services are likely responsible for nearly fivefold reduction in infant and child mortality in the United States [3]. Knowledge and understanding of current situation of PICUs is the first step for future planning, development, or expansion of PICUs in any country. Survey reports regarding the PICU structure, staffing, and characteristics are available from countries like USA [4], Spain [2], and Turkey [5].

Pediatric Critical Care Medicine (PCCM) is a relatively a new subspecialty of pediatrics in Pakistan. There is a paucity of data available regarding PICU structure, organization, and staffing from developing countries like Pakistan. The objective of this study is to describe the structure, staffing resources, equipment, academic activities, and characteristics of pediatric population of PICUs across the country.

\section{Methods}

This was a descriptive observational survey. PICU was described as a separate physical facility or unit specifically designated for the treatment of pediatric patients who, because of shock, trauma, or other life-threatening conditions, require intensified, comprehensive observation and care [6]. Questionnaire used for this survey was adapted from Randolph et al. [4] with permission. The questionnaire that sought information addressing the 5 components of a PICU, that is, structure/organization, personal details, equipment/technical support, academics/Research, and patient characteristics/outcome was emailed to the director of each unit Telephone follow-up was attempted to get maximal responses, if the survey was not returned after four weeks. All requested 
TABLE 1: Characteristics of PICUs.

\begin{tabular}{lc}
\hline General & \\
Number of hospitals & 16 \\
Number of pediatric beds & 2551 \\
Number of PICU beds & 155 \\
Tertiary care center & $16 / 16$ \\
Public/private & $13 / 3$ \\
Types of PICUs & \\
Medical & $12(75 \%)$ \\
Cardiac & $3(18.7 \%)$ \\
Multidisciplinary & $1(6.2 \%)$ \\
Sizes of PICUs (no. of beds) & \\
$<5$ & $1(6.2 \%)$ \\
06-10 & $7(43.8 \%)$ \\
$>11$ & $8(50 \%)$ \\
Pediatric subspecialties & \\
Pediatric surgery & $13(81.2 \%)$ \\
Pediatric cardiology & $10(62.5 \%)$ \\
Pediatric nephrology & $8(50 \%)$ \\
Pediatric neurology & $6(37.5 \%)$ \\
Pediatric hem-onc & $8(50 \%)$ \\
Equipment/technology & \\
Cardiac monitors & $16(100 \%)$ \\
Mechanical ventilators & $15(93.8 \%)$ \\
Crash carts & $14(87.5 \%)$ \\
Infusion pumps & $15(93.8 \%)$ \\
\hline & $8(50 \%)$ \\
\hline
\end{tabular}

information of questionnaire about the medical activity during 2009 was collected from PICUs of these hospitals. Survey administration occurred from January through June 2010. Adult ICUs providing pediatric care children, neonatal intensive care units, high dependency units, and services not fulfilling the PICU definition for the study were excluded. The estimated pediatric population under 14 years of age was 72.4 million defined by the Pakistan census for 2010, which was $40 \%$ of total population [7]. The number of PICU beds per pediatric population was sum of children divided by the number of PICU beds.

The study was approved by the Ethical Review Committee of The Aga Khan University Hospital (Ref. no. 1594PedERC). SPSS version 16 was used for analysis of descriptive statistics.

\section{Results}

We obtained a $100 \%$ response rate. We identified 16 PICUs at different hospitals all over Pakistan.

3.1. Structure/Organization of PICUs. All were tertiary care teaching hospitals affiliated with medical colleges of which $3 / 16$ were pure cardiac intensive care units (CICU). (Table 1 ) A total of 155 PICU beds were identified constituting in all

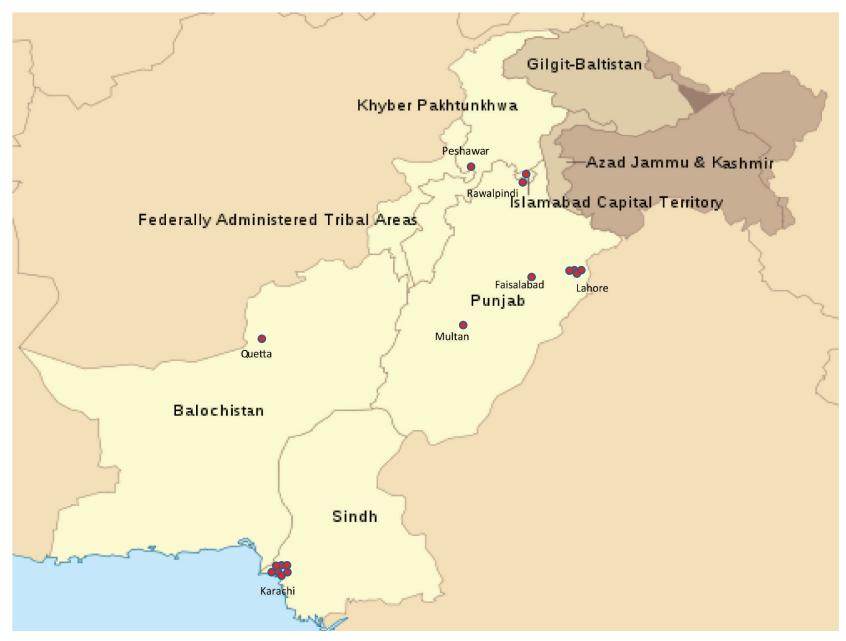

FIGURE 1: The locations of PICUs by province: each dot represents one PICU.

these hospitals with mean number of bed per PICUs was 9.7 (min 4, max 28). Thus the average numbers of PICU beds per pediatric population were 1/500,000 children under 14 years. As the total pediatric beds in hospitals were approximately 2551 , so the PICUs constitute only $6 \%$ of hospital beds. Majority 12 (75\%) of PICUs were located in three large cities, that is, Karachi, Lahore, and Rawalpindi (Figure 1).

3.2. Personal. All PICUs had a full-time medical director. Most of them (medical and multidisciplinary PICUs) were closed units run by pediatricians. Cardiac and surgical units were managed by anesthesiologists. There were only 3 out of 16 pediatricians (19\%) who had advanced training in pediatric critical care medicine. One has done three-year training from accredited pediatric critical care medicine fellowship and board certified in pediatric critical care medicine from the American Board of Pediatrics, All nurses had bachelor degree or diploma in nursing and special training for PICUs, and only 6 (37\%) PICUs had a nurse to patient ratio of $1: 1$ or $1: 2$. Availability of various subspecialties in different PICU was variable (Table 1).

3.3. Equipment/Technical Support. All units had cardiac monitoring and pulse oximetry for each bed with crash cart and mechanical ventilation available in almost all PICUs (Table 1) Invasive hemodynamic monitoring in critically ill patients was available in $8(50 \%)$ of the PICUs with only one PICU having availability of advance technology like High Frequency Oscillator Ventilator (HFOV), Continuous Renal Replacement Therapy (CRRT), Therapeutic aphaeresis, and Intracranial Pressure (ICP) monitoring.

3.4. Academic/Research. All units had the capacity of maintaining patient census. Most of PICUs had post-graduatetraining program for pediatric medicine for residents with a rotation of 3-6 months during which they are taught common critical care problems along with basic principles of critical care. Only one program (AKU) offered a 2-year fellowship in pediatric critical care medicine after completion 
of 4-year training in pediatrics, and only one institution offered American Heart Association sponsored Basic Life Support and Pediatric Advance Life Support courses for all health care providers (physicians and nurses). Eleven (69\%) of PICUs had protocol/guidelines for recognition and treatment of pediatric common critical illness.

3.5. Patient Characteristics and Outcome. A cumulative of 7,376 admissions (6181 to medical/multidisciplinary units and 1195 to cardiac units postoperatively) were recorded in all the PICUs during the calendar year of 2009. Neurological illnesses, cardiovascular pathology, respiratory ailment, and sepsis were the most common causes of admission to the medical units. Only 4 hospitals managed postoperative cardiac patients. Repair of tetralogy of Fallot, ventricular \& atrial septal defects, coarctation of aorta, and arterial switch operation were the most common cardiac procedures performed and subsequently managed in these units. Mortality in cardiac intensive care units was 6.6\% (79/1195). The overall mortality in all the PICUs was $12 \%$ ( $\min 4 \%-\max 29 \%$ ).

\section{Discussions}

To our knowledge, this is first basic report regarding structure, organization and staffing of PICUs in Pakistan. Our reports revealed that PICUs in Pakistan are scarce, understaffed, have limited equipment, and have limited formal training structure. Most of our observations are like data reported from Turkey [5]. There were similar problems have been published from ICUs in other less developed countries like Croatia [8], Mongolia [9] and Sri Lanka [10]. One in every 11 children in Pakistan dies before reaching his or her 5th birthday. Sepsis-related illness, diarrhea and pneumonia are the leading cause of death in children under-five [11]. These deaths are largely related to delay in seeking medical advice and lack of basic intensive care therapy like oxygen, positive pressure ventilation, rapid intravenous fluid resuscitation, inotropes, and antibiotics. The availability of designated PICUS providing basic pediatric intensive care interventions to these acutely ill or injured children will significantly improve their survival rates [12]. With a current pediatric population in Pakistan under 14 years [9], this translates into only 1 PICU bed for approximately 500,000 children under 14 years as compared to 1 bed/10,000 children in USA and 1/1000 in the UK $[4,13]$ It has been previously reported from Spain [2], Turkey [5] and Brazil [14] there was uneven geographical distribution of PICUs in these countries. Similarly, the most of the PICUs were located in three major cities of Pakistan as shown in Figure 1, and lack of retrieval services may lead to delay in presentation, thus increasing the chances of complications and mortality. There is no centralization or regionalization to decrease mortality and length of stay by increasing the volume of patients like in UK [15]. Our survey also demonstrated that apart from structure and distribution of the limited PICUs beds, staffing of these units was another major issue.

Majority of the units were managed by pediatricians out of whom only $19 \%$ had any special training in pediatric intensive care medicine. Only $37 \%$ of PICUs had a nurse to bed ratio of $1: 1$ or $1: 2$, and these numbers are substantially low when compared to other countries [16]. Long duty hours and low salaries lead to a low morale and decreased job satisfaction among staff [17].

To improve the outcome of critically ill patients, the availability of advance equipment with appropriate technical support is essential [18]. It was encouraging to find that most centers were equipped with basic intensive care equipment, that is, mechanical ventilators and crash carts. When it came to advance equipment required for management of complex diseases, the picture was dismal. Only one center, a private sector hospital had the capacity of managing pediatric patients requiring advanced therapies like HOFV, CRRT or therapeutic apheresis.

The major medical admission diagnostic categories of our PICUs given in this report are similar to those seen in PICUs of developed countries. Most of PICUs in developed countries are multidisciplinary (combined medical and surgical cases) and are involved in the postoperative care of children $[2,4]$. This was not the case in our survey where only 1 PICU is fulfilled to definition of a multidisciplinary unit. Our report revealed that approximately 1200 postcardiac surgical cases were cared in four pediatric cardiac intensive care units with mortality rate of $6.6 \%$ percent during the calendar year. Our pediatric cardiac intensive care units (PCICUs) are in evolving phase and have rapidly grown for perioperative care of children with congenital heart disease over the last decade. Interestingly similar to other reports [19], pediatric neurological illnesses requiring intensive care management were seen frequently in our survey too.

Previous reports have shown that the implementation of intensivist-led team services and fellowship training programs improved survival, decreased length of stay, and resource utilization [20, 21]. Additionally, regular education and training of in-service pediatric residents on common pediatric critical care problems has been shown to increase short-term knowledge [20,22]. Our survey showed that there was only one academic institution offering fellowship training in pediatric critical care medicine for whole country and few hospitals had educational curriculum for residents rotating in the PICUs. Implementation of protocols in the management of critically ill patients has been shown to improve the outcome [21]. Though 69\% PICUs reported use of protocols for management of common acute critical illnesses, mechanism of protocol updates was not reported [23].

There are several limitations in our study. We did not define the level of care in different PICUs, categorize admissions on patient's age, gender, and social background, and determine the bed occupancy and length of stay in this survey. Our data may not exactly reflect the total pediatric intensive care services in our country as we have not included children who are cared in neonatal and adult intensive care units. We were unable to collect the data regarding infection control policy of our PICUs. Data reflected in this survey were self-reported by the PICU directors and were not subjected to verification. Our report lacks assessment of PICU's demand.

In conclusion, facilities to manage pediatric patients requiring intensive care are extremely limited. The available units have a skewed distribution making accessibility to the general 
population difficult leading to late presentation and worse outcomes. These units are understaffed and ill equipped to manage critically ill pediatric patients. There is a significant need for academic PICU programs to address this problem.

\section{Appendix}

The following Pediatric Intensive Care Units took part in the study:

(1) Institute of Child Health and Children's Hospital, Lahore (M.H. Hamid), Children Hospital,

(2) Pakistan Institute of Islamabad (A. Fareedi),

(3) Mayo Hospital, Lahore (A. Sultan),

(4) Sheikh Zayed Hospital, Lahore (W. Hussain),

(5) Ittefaq Hospital, Lahore (M.A. Khan),

(6) Allied Hospital, Faisalabad (A. Butt),

(7) Children Hospital Complex and Institute of Child Health, Multan (A. Khurshid)

(8) Armed Forces Institute of Cardiology and National Institute of Heart Diseases (S. Naqvi),

(9) Aga Khan University Hospital, Karachi (A. Haque),

(10) Civil Hospital, Karachi (F. Arif),

(11) Liaquat National Hospital, Karachi (S. Qamar),

(12) Abbasi Shaheed Hospital, Karachi (S. Mirza),

(13) National Institutes of Cardiovascular Disease, Karachi (N. Amjad),

(14) National Institute of Child Health, Karachi (M. Fareeduddin),

(15) Khyber Teaching Hospital, Peshawar (N.R. Kazmi),

(16) Children's Hospital, Quetta (D. Qureshi).

\section{Conflict of Interests}

The authors declared that there is no conflict of interests.

\section{References}

[1] D. Epstein and J. E. Brill, "A history of pediatric critical care medicine," Pediatric Research, vol. 58, no. 5, pp. 987-996, 2005.

[2] J. López-Herce, L. Sancho, and J. M. Martinón, "Study of paediatric intensive care units in Spain," Intensive Care Medicine, vol. 26, no. 1, pp. 62-68, 2000.

[3] J. A. Carcillo, "What's new in pediatric intensive care," Critical Care Medicine, vol. 34, supplement 9, pp. S183-S190, 2006.

[4] A. G. Randolph, C. A. Gonzales, L. Cortellini, and T. S. Yeh, "Growth of pediatric intensive care units in the United States from 1995 to 2001," Journal of Pediatrics, vol. 144, no. 6, pp. 792798, 2004.

[5] T. F. Köroğlu, S. Atasever, and M. Duman, "A survey of pediatric intensive care services in Turkey," Turkish Journal of Pediatrics, vol. 50, no. 1, pp. 12-17, 2008.
[6] Committee on Hospital Care of the American Academy of Pediatrics and Pediatric Section of the Society of Critical Care Medicine, "Guidelines and levels of care for pediatric intensive care units," Pediatrics, vol. 92, no. 1, pp. 166-175, 1993.

[7] "Population by selective age groups," Population Census Organisation, Government of Pakistan, 2013, http://www.census.gov .pk/SelectiveAge.htm.

[8] V. Degoricija, S. Šefer, M. Kujundžić-Tiljak, and M. Gjurašin, "Intensive care units in Croatia: 2001 Survey," Croatian Medical Journal, vol. 43, no. 6, pp. 713-721, 2002.

[9] M. W. Dünser, O. Bataar, G. Tsenddorj, G. Lundeg, S. Jochberger, and S. Jakob, "Intensive care medicine in Mongolia's 3 largest cities: outlining the needs," Journal of Critical Care, vol. 24, no. 3, pp. 469.el-469.e6, 2009.

[10] A. B. Yatawatte, C. R. Wanniarachchi, and C. D. Goonasekera, "An audit of state sector intensive care services in Sri Lanka," The Ceylon Medical Journal, vol. 49, no. 2, pp. 51-54, 2004.

[11] L. Liu, H. L. Johnson, S. Cousens et al., "Global, regional, and national causes of child mortality: an updated systematic analysis for 2010 with time trends since 2000," The Lancet, vol. 379, no. 9832 , pp. 2151-2161, 2012.

[12] S. Singhi and N. Niranjan Kissoon, "Pediatric intensive care in India: why, how and role of global collaboration!", Indian Journal of Pediatrics, vol. 75, no. 6, pp. 589-590, 2008.

[13] B. Maybloom, J. Chapple, and L. L. Davidson, "Admissions for critically ill children: where and why?" Intensive and Critical Care Nursing, vol. 18, no. 3, pp. 151-161, 2002.

[14] D. C. de Souza, E. J. Troster, W. B. de Carvalho, S. H. Shin, and A. M. G. Cordeiro, "Availability of pediatric and neonatal intensive care units in the city of São Paulo," Jornal de Pediatria, vol. 80, no. 6, pp. 453-460, 2004.

[15] G. Pearson, P. Barry, C. Timmins, J. Stickley, and M. Hocking, "Changes in the profile of paediatric intensive care associated with centralisation," Intensive Care Medicine, vol. 27, no. 10, pp. 1670-1673, 2001.

[16] F. O. Odetola, S. J. Clark, G. L. Freed, S. L. Bratton, and M. M. Davis, "A national survey of pediatric critical care resources in the United States," Pediatrics, vol. 115, no. 4, pp. e382-e386, 2005.

[17] M. W. Dünser, I. Baelani, and L. Ganbold, "A review and analysis of intensive care medicine in the least developed countries," Critical Care Medicine, vol. 34, no. 4, pp. 1234-1242, 2006.

[18] P. G. Bastos, W. A. Knaus, J. E. Zimmerman, A. Magalhães Jr., X. Sun, and D. P. Wagner, "The importance of technology for achieving superior outcomes from intensive care," Intensive Care Medicine, vol. 22, no. 7, pp. 664-669, 1996.

[19] R. C. Tasker, "Pediatric neurocritical care: is it time to come of age?" Current Opinion in Pediatrics, vol. 21, no. 6, pp. 724-730, 2009.

[20] F. O. Odetola, A. L. Rosenberg, M. M. Davis, S. J. Clark, R. E. Dechert, and T. P. Shanley, "Do outcomes vary according to the source of admission to the pediatric intensive care unit?" Pediatric Critical Care Medicine, vol. 9, no. 1, pp. 20-25, 2008.

[21] M. M. Pollack, K. M. Patel, and U. E. Ruttimann, "Pediatric critical care training programs have a positive effect on pediatric intensive care mortality," Critical Care Medicine, vol. 25, no. 10, pp. 1637-1642, 1997.

[22] A. Haque, R. Haider, and M. S. Salat, "Teaching paediatric criical care medicine to paediatric residents," Journal of the Pakistan Medical Association, vol. 60, no. 4, pp. 319-321, 2010.

[23] J. J. Hammond, "Protocols and guidelines in critical care: development and implementation," Current Opinion in Critical Care, vol. 7, no. 6, pp. 464-468, 2001. 


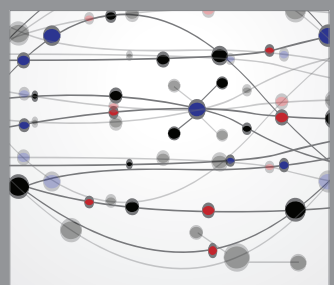

The Scientific World Journal
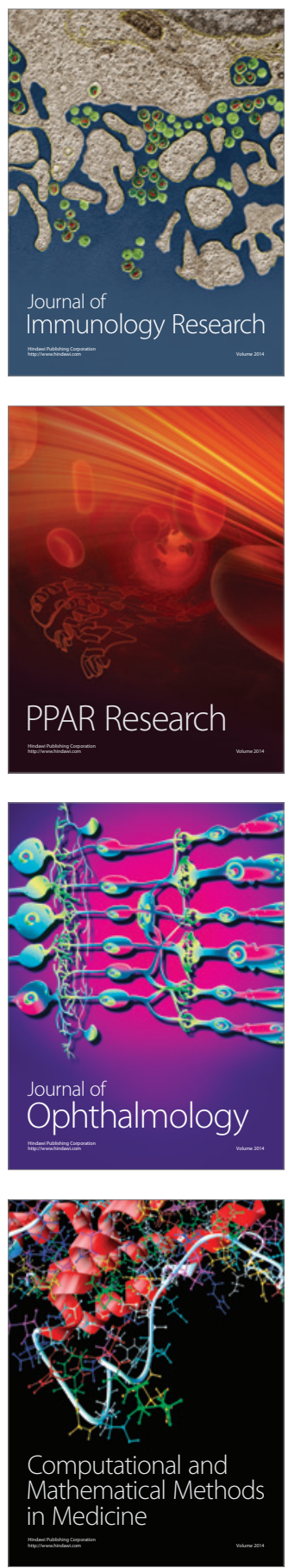

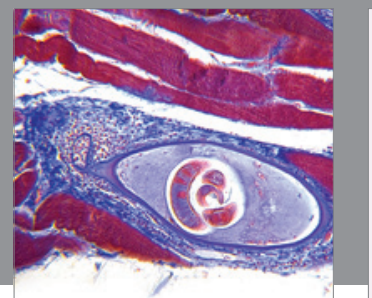

Gastroenterology

Research and Practice
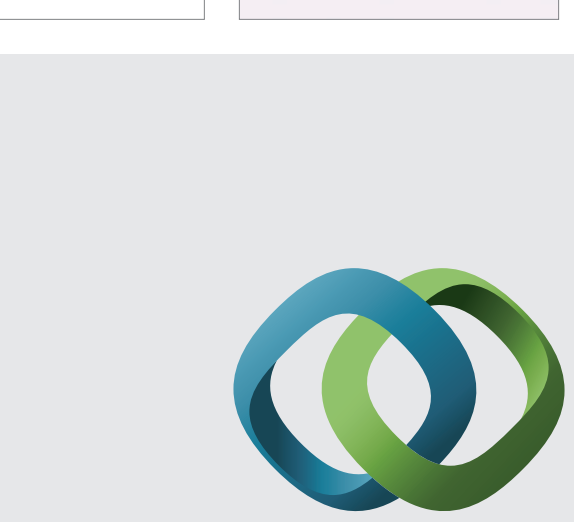

\section{Hindawi}

Submit your manuscripts at

http://www.hindawi.com
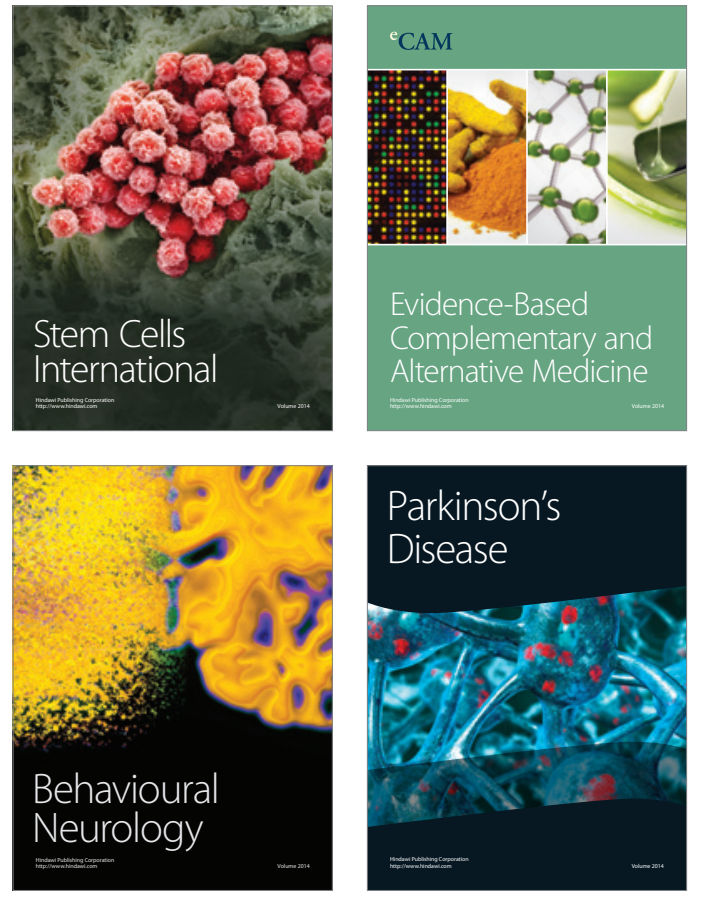
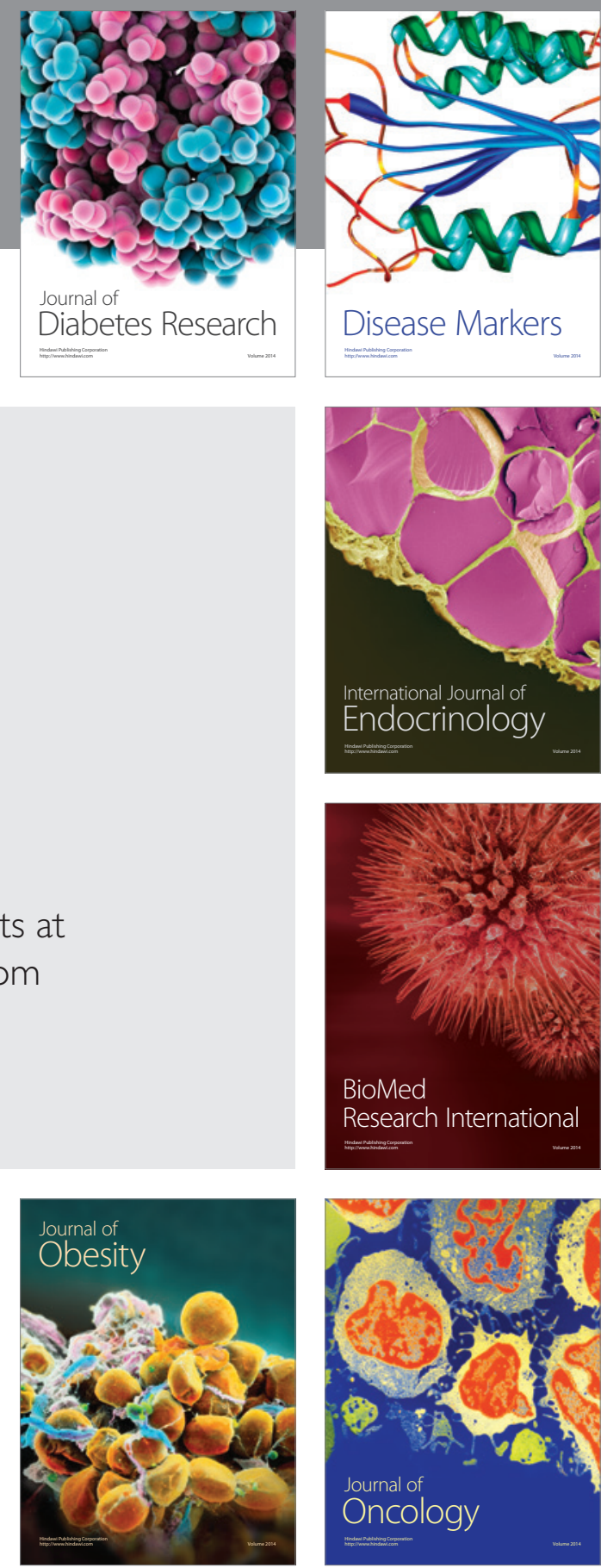

Disease Markers
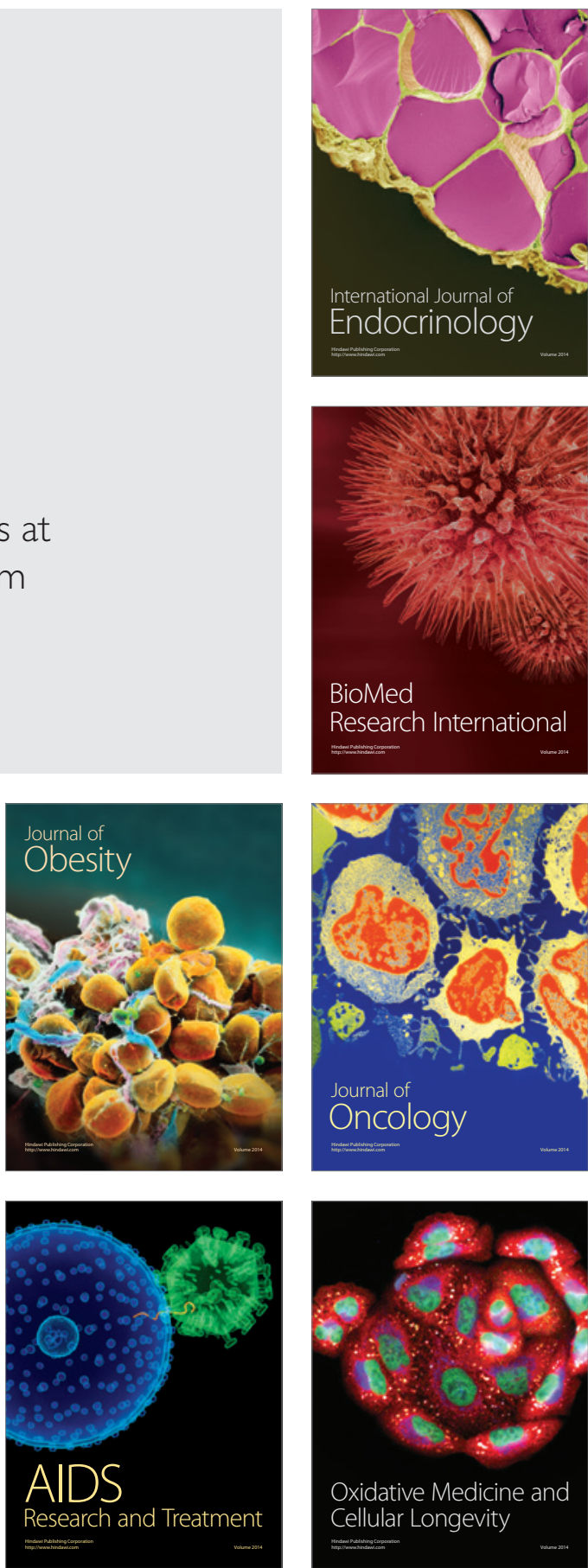